\title{
PRECIOS DE ACTIVOS Y POLÍTICA MONETARIA EN LA NUEVA SÍNTESIS NEOCLÁSICA
}

\section{Ignacio Perrotini Hernández*}

División de Estudios de Posgrado de la Facultad de Economía,

Universidad Nacional Autónoma de México

(Recibido 16 agosto de 2013, aceptado 18 de septiembre de 2013)

I can calculate the motion of heavenly

bodies, but no the madness of people.

Isaac Newton

\section{Resumen}

La cuestión de si un banco central que opera con el marco de política monetaria de objetivo de inflación postulado por la Nueva Síntesis Neoclásica (NSN) debe reaccionar ante los movimientos de los precios de los activos ha sido objeto de un debate teórico. En el presente artículo explicamos las principales posiciones teóricas relativas a este problema fundamental y el modelo canónico de la NSN, evaluamos las dos hipótesis principales esgrimidas en la polémica, discutimos las ventajas y desventajas de cada una y concluimos que la tasa de interés no es suficiente para evitar la exuberancia irracional y los ciclos boom-bust en los mercados de activos.

\begin{abstract}
The paper is aimed at assessing the theoretical debate as to whether an inflation-targetingcentral-bank should react to asset price movements. The main theoretical tenets of the debate, the canonical model of the New Neoclassical Synthesis and the two main competing hypotheses of the debate are both explained and discussed. By way of conclusion, it is argued that the rate of interest is not a suitable policy instrument for preventing irrational exuberance and boom-bust cycles in the asset markets.
\end{abstract}

Clasificación JEL: G12, E52, E58

Palabras clave: Valuacion de Activos, Política Monetaria, Banco Central.

* Profesor de Teoría y Política Monetaria, Divisón de Estudios de Posgrado de la Facultad de Economía de la UNAM, Ciudad Universitaria, México, D.F. C.P. 04510. Correo electrónico: iph@.unam.mx 


\section{Introducción}

Los mercados financieros internacionales han experimentado profundos cambios recientemente. Estas mutaciones incluyen innovaciones en los instrumentos de ahorro y derivados de capital, en los métodos de colocación y negociación de los títulos y en las formas de prorrateo y transferencia de los riesgos de capital, de los intereses y dividendos.

En periodos normales del ciclo económico la innovación financiera contribuye notablemente a la estabilidad y a la eficiencia de los mercados financieros porque abate los costos de operación, contribuye a amortiguar los choques adversos al incrementar la liquidez de los mercados y propicia la asignación racional de los recursos y el acopio de capital de riesgo para el desarrollo tanto a nivel micro (empresas financieras y no financieras, hogares) como en el aspecto macroeconómico (Bank for International Settlements 1994, Cohen 1996, Hernández Trillo 2003:30-49). En efecto, los nuevos instrumentos financieros como los futuros y las opciones ${ }^{1}$ añaden nueva información a los precios de los títulos (Cohen 1996:22), ayudan a disminuir y diseminar el riesgo asociado al incremento del crédito bancario al fortalecer la integración de los mercados, aprontando a disposición de los inversionistas instrumentos que pulverizan el riesgo, amplían las coberturas y facilitan a bancos y operadores la posibilidad de desplegar actividades sintéticas que permiten la configuración de portafolios integrados por títulos con operaciones de futuros, swaps y opciones.

Asimismo, la desregulación y la mayor integración de los mercados (Kane 1991, Allen y Gale 2009, Dewatripont et al. 2010, Tirole 2010), la propagación de la innovación financiera, la liberalización de las cuentas de capitales (Davis 1995:97), el avance de la telemática en el mercado global (Arcelli 1996, Guttmann 2003, Fullenkamp y Nsouli 2004) y el impacto del alud de información disponible ipso facto con costos decrecientes a través de los medios cibernéticos en las expectativas de los operadores (Bank for International Settlements 2000, Goodhart 2000) han acrecentado en ocasiones la volatilidad de los precios de los activos financieros. Las transacciones cotidianas en los sistemas de pagos así como los movimientos de fondos de corto plazo y de títulos de deuda soberana se han redimensionado exponencialmente en el curso de unos cuantos años. La volatilidad de las tasas de interés y el predominio del régimen de tipo de cambio flotante -adoptado generalmente desde el colapso del régimen de tipo de cambio fijo de Bretton Woods- se hallan a la base de este crecimiento exponencial. Por ejemplo, según datos de la Fed (2012) el volumen promedio diario de pagos en dólares realizados a través del Fedwire (sistema de pagos bruto en tiempo real de la Fed) fue equivalente al 17 por ciento del PIB de Estados Unidos en 2011, mientras que ya en 2001 el valor nocional de los derivados negociados en el mercado mundial había ascendido a 139.7 trillones de dólares (Bank for International Settlements 2001).

Esta notable expansión del capital financiero en ocasiones ha conducido a espasmos de inestabilidad de la economía real de varios países, a episodios

\footnotetext{
1 Los futuros más socorridos en el presente son los interest rate futures, foreign currency futures y stock index futures; las opciones, a su vez, otorgan el derecho de compra o venta de un instrumento financiero a un precio prefijado con fecha de vencimiento preestablecida.
} 
de fragilidad financiera (Bernanke y Gertler 1990, Davis 1995, Allen y Gale 2000, 2009), y aún a crisis financieras y depresiones económicas de gran calado (Orhangazi 2008, Tirole 2010, Duménil y Levy 2011). En el Annual Report of the Bank for International Settlements de 2001 se sostiene que la liberalización de los mercados financieros "ha incrementado el espectro de pronunciados ciclos financieros" y que "el daño causado por la inestabilidad financiera ha sido particularmente severo en los países de economías emergentes" (Bank for International Settlements 2001:123). El desequilibrio de los precios de los activos, especialmente en el caso de burbujas prolongadas que colapsan precipitadamente, suele afectar al consumo, la inversión y a la demanda agregada. Por tanto, parece pertinente analizar la cuestión de si las fluctuaciones de los precios de los activos influyen en el modus operandi de la política monetaria del banco central.

El crecimiento exponencial de los nuevos instrumentos financieros derivados de capital- no implica inmediata ni necesariamente que el poder de los bancos centrales para controlar la inflación se evapore. Sin embargo, es evidente que el desarrollo de la innovación financiera altera el contexto en que operan los bancos centrales, modifica los canales de transmisión de la política monetaria, la importancia de los indicadores monetarios y la eficacia de los instrumentos de la política de intervención del banco central (Vrolijk 1997). De suerte que es prudente no perder de vista que la dialéctica de los mercados de activos ejerce una influencia no lineal -estabilizadora unas veces y desestabilizante en otras- sobre los precios de los activos, efecto dual identificado al menos hace dos decenios por el Bank for International Settlements (1994:3): "En condiciones normales, por tanto, es probable que los mercados de derivados ejerzan una influencia estabilizante sobre los títulos subyacentes. Sin embargo, en periodos de tensiones, los mercados de derivados pueden exacerbar la volatilidad de los precios de los mercados financieros en el corto plazo". En consecuencia, el imperativo de la política monetaria de estabilizar los precios y las expectativas se halla en razón directa al desarrollo de los mercados financieros.

Una cuestión toral del debate monetario contemporáneo es si la meta de inflación debe contemplar las variaciones de los precios de los activos. Por una parte, dada la mayor liquidez de estos mercados vis-à-vis los recursos de la autoridad monetaria, las decisiones de los agentes privados pueden neutralizar la efectividad de las acciones (por ejemplo, operaciones de mercado abierto) del banco central y, por otra, precisamente, "en periodos de tensiones" un desequilibrio en los precios de los activos se transmite con mayor rapidez a través de la urdimbre tejida entre los mercados, urdimbre construida en parte gracias a la innovación financiera. El hecho de que la evolución reciente de los mercados financieros ha tendido a incrementar la sensibilidad de los mercados financieros respecto de las fluctuaciones de las tasa de interés y de los tipos de cambio, torna ineludible la cuestión de si la regla de política monetaria debe incluir la inflación de los precios de los activos, es decir, la cuestión de si los bancos centrales deben reaccionar ante los movimientos de los precios de los activos. En el presente artículo nos proponemos dar cuenta de las principales posiciones teóricas relativas a esta cuestión fundamental. En la segunda sección, exponemos los elementos teóricos esenciales del modelo de política monetaria de la Nueva Síntesis Neoclásica (NSN), en la tercera evaluamos los postulados de las dos hipótesis sobre la bondad de incluir o excluir los precios de los activos en 
la regla monetaria óptima y en la sección final presentamos un breve balance del debate y algunas aporías y reflexiones críticas acerca de los méritos relativos del instrumento de política monetaria, la tasa de interés, para evitar la exuberancia irracional y los ciclos boom-bust en los mercados de activos.

\section{El Modelo Canónico de la Nueva Síntesis Neoclásica}

El modelo de la NSN es un marco de política monetaria de objetivos de inflación (inflation targeting, IT en adelante) que consiste en una simbiosis -una nueva síntesis- de la hipótesis de expectativas racionales y los postulados de la antigua síntesis neoclásica "keynesiana" de la segunda postguerra. La estrategia IT es el marco de política monetaria más popular en la actualidad, un gran número de países operan con algún tipo de IT fijo o flexible. ${ }^{2}$

Las características principales de este enfoque de política monetaria son: i) la declaración explícita de metas de inflación para un periodo determinado; ii) la adopción de una tasa inflación baja y estable como el objetivo principal; iii) la transparencia, credibilidad y rendición de cuentas de las acciones del banco central; iv) la ausencia de metas intermedias (por ejemplo, metas de tipo de cambio o de expansión monetaria); v) la tasa de interés es el instrumento de la política monetaria y el principal canal de transmisión de los efectos en la demanda agregada para controlar la inflación (Taylor 1993, Bernanke et al. 1999, Woodford 2003); vi) un régimen de tipo de cambio flexible determinado por la paridad de tasas de interés (cf. Bernanke y Mishkin 1997, Svensson 1998, Clarida et al. 1997, Bernanke et al. 1999, Woodford 2003, Arestis 2007).

La estructura del modelo consiste en tres ecuaciones interrelacionadas que representan la dinámica de la demanda agregada (curva IS), la inflación (curva de Phillips) y la tasa de interés real, que es la función de reacción del Banco Central:

$$
\begin{gathered}
y_{t}=y_{0}-\alpha r+\varepsilon_{1} \\
\pi_{t}=\pi_{t-1}+\beta\left(y_{t}-y^{*}\right)+\varepsilon_{2} \\
\left.r_{t}=r *+\phi_{\pi} \pi_{\Gamma}+\phi_{y} y_{\Gamma}, \phi\right\rangle 0
\end{gathered}
$$

donde $r$ es la tasa de interés real, $y_{0}$ es el ingreso autónomo, $y_{t}$ es el nivel de ingreso observado en el periodo $t, y^{*}$ es el ingreso potencial, $\pi_{t}$ es la inflación observada, $\pi_{t-1}$ es la inflación rezagada un periodo, $y_{\Gamma}=\left(y_{t}-y^{*}\right)$ es la brecha de producto, $\pi^{*}$ es el objetivo de inflación, $\pi_{\Gamma}=\left(\pi_{t}-\pi^{*}\right)$ es la brecha de inflación, $\mathrm{r}^{*}$ es la tasa natural de interés, $\varepsilon_{1}$ y $\varepsilon_{2}$ son perturbaciones o choques aleatorios. La curva de Phillips expresa la influencia de las expectativas de los agentes económicos en la inflación: $y_{\Gamma}$ se relaciona con la dinámica de la inflación, razón por la cual la tasa de interés se ajusta de acuerdo con las expectativas sobre $y_{\Gamma}$. Si $y_{\Gamma}$ aumenta se incrementa la inflación, y con ella aumenta también $\pi_{\Gamma}$. En consecuencia, la autoridad monetaria debe aumentar la tasa de interés

\footnotetext{
2 El esquema IT ha sido adoptado implícita o explícitamente en diversas formas por los bancos centrales de los siguientes países: Australia, Brasil, Canadá, Chile, Colombia, Corea del Sur, Estados Unidos, Guatemala, Islandia, Israel, México, Noruega, Nueva Zelanda, Polonia, Reino Unido, República Checa, Sudáfrica, Suecia, Suiza, Tailandia y la Unión Monetaria Europea (Bernanke et al. 2009, Perrotini 2011).
} 
$r_{t}$. Conforme aumenta $r_{t}$ la inflación disminuye, $\pi_{t}$ y $y_{t}$ tenderán hacia $\pi^{*}$ y $y^{*}$ respectivamente $\mathrm{y}$, por tanto, las brechas de inflación y de producto tenderán a cero, $\pi_{\Gamma}=0$ y $y_{\Gamma}=0$. En el límite, $r_{t}=r^{*}$, es decir, la tasa de interés real actual se igualará con la tasa natural de interés y se realizará el objetivo de inflación $\pi^{*}$. Al fijarse la tasa nominal de corto plazo, se fija también la tasa de interés real $r_{t}$ mediante la diferencia $i_{t}-\pi^{\varepsilon}$, i.e., restando las expectativas de inflación a la tasa de interés nominal. De suerte que se asegura la convergencia entre $r_{t}$ y $r^{*}$ y así el Banco Central puede controlar la inflación sobre la base de la manipulación de las expectativas de demanda agregada.

Es evidente que los precios de los activos no desempeñan ningún papel en el marco de política monetaria IT. Las extensiones de este modelo realizadas por Ball (1999) y Svensson (1999) que incluyen al tipo de cambio como un canal adicional de transmisión de los choques estructurales y de la política monetaria son apenas dos excepciones en elmare magnum de literatura que excluye a los precios de los activos de la función de reacción del banco central. En una economía abierta, es necesario incorporar el efecto de las variaciones del tipo de cambio (e) en la demanda agregada y en la inflación. Ball (1999) y Svensson (1999) mostraron que existe un vínculo directo entre la tasa de interés real y el tipo de cambio:

$$
e_{t}=\psi r_{t}+\varepsilon_{3}, \psi>0
$$

$\psi$ mide la relación entre el tipo de cambio y la tasa de interés real y $\varepsilon_{3}$ es un término de error aleatorio; si $\psi=1$ entonces el tipo de cambio se rige por la condición de paridad descubierta de tasas de interés, i.e., la paridad entre la tasa de interés nacional $(i)$ y la internacional $\left(i^{*}\right)$. Si se consideran las expectativas de tipo de cambio, la relación entre éste y la paridad descubierta de tasas de interés es como sigue:

$$
e_{t}-E\left(e_{t+1}\right)=i_{t}-i^{*}+\varepsilon_{t}
$$

En el modelo NSN se supone que se satisface la paridad descubierta de tasas de interés. Por tanto, el tipo de cambio responde a esa condición. Así, en el largo plazo el papel del tipo de cambio en el modelo NSN se desvanece a medida que con la disminución de la inflación el traspaso de las fluctuaciones del tipo de cambio a los precios se elimina.

De lo anterior, se infieren las siguientes tesis principales del modelo NSN: 1) la inflación es un fenómeno monetario; 2) la estabilidad de precios es la meta primordial de la política monetaria que resulta de una tasa de interés de largo plazo neutral (Bernanke et al. 1999, Bernanke y Mishkin 1997, Woodford 2003), 3) la tasa de interés es el único instrumento de la autoridad monetaria que ancla la inflación (Bernanke et al. 1999, Taylor 1999, Woodford 2003) y 4) el tipo de cambio se determina mediante la paridad de tasas de interés y, al final del día, no desempeña ningún papel en el marco de política monetaria.

El supuesto de que la relación de causalidad es de la tasa de interés al tipo de cambio significa que no existe un canal de transmisión del tipo de cambio, que los flujos de capital tienen una extraordinaria elasticidad respecto de la paridad descubierta de tasas de interés y, por tanto, la tasa de interés -el único instrumento de la política monetaria del banco central- controla la demanda agregada, la inflación y el tipo de cambio. Por esta razón, en la teoría de la 
NSN se concluye que el banco central no debe reaccionar ante los movimientos de los precios de los activos ni hace falta que la función de reacción incorpore un coeficiente significativo para sopesar el efecto de la inflación de los mercados de activos (Bernanke y Gertler 1999, 2001, Mishkin 2001). La única excepción a este principio que se admite es cuando las fluctuaciones de los precios de los activos modifican las expectativas de los agentes privados, lo cual sucede como resultado de un cambio en los fundamentales del mercado, por ejemplo, una política monetaria "completamente óptima" deberá acomodar los cambios en los precios de los activos debido a burbujas temporales o a choques tecnológicos que modifican la tasa natural de interés (Bernanke y Gertler 2001:256).

No obstante, con base en evidencia empírica diversos analistas sostienen que varios bancos centrales sistemáticamente realizan intervenciones esterilizadas en los mercados de cambios en el marco de política monetaria IT, y que estas intervenciones esterilizadas sí han sido efectivas para la consecución del objetivo de inflación (Bofinger y Wollmershäuser 2001, Frenkel et al. 2003, Hüfner 2004, Mántey 2009, Capraro y Perrotini 2011). Por tanto, concluyen, estas intervenciones constituyen un segundo instrumento regular e independiente de la política monetaria, lo cual explica el fenómeno conocido como "miedo a flota" el tipo de cambio (Calvo y Reinhart, 2002).

Si se admite que: i) las intervenciones en los mercados de cambio operan como un canal de transmisión de la política monetaria adicional, ii) las variaciones en los precios de los bonos son en esencia resultado de cambios en las tasas de interés que afectan las hojas de balance y el gasto en consumo e inversión y iii) la política monetaria afecta el precio de las acciones bursátiles domésticas y al tipo de cambio provocando alteraciones en el valor real de la riqueza de los residentes nacionales, entonces la cuestión de si los bancos centrales deben reaccionar frente a los movimientos de los precios de los activos amerita una inspección circunspecta.

\section{Precios de activos y Banca Central}

La cuestión de si los precios de los activos deben ser parte de la formulación de la política monetaria ha sido objeto de un nutrido debate fundamentalmente entre dos posiciones cuyos principales exponentes son, por una parte, Bernanke y Gertler $(1999,2001)$ y, por otra, Cecchetti, Genberg, Lipsky y Wadhwani $(2000,2002){ }^{3}$ Mientras Bernanke y Gertler consideran que la regla de política monetaria del banco central no debe incluir los precios de los activos (bonos, acciones bursátiles, títulos, tipo de cambio, bienes raíces, etc.), Cecchetti y sus coautores afirman que la autoridad monetaria conseguiría mejor aún sus objetivos si reaccionara ante los movimientos de los precios de los activos. A continuación glosamos y comentamos las tesis centrales de ambos enfoques.

Bernanke y Gertler $(1999,2001)$ elaboran un modelo dinámico de una economía con tres sectores (hogares que consumen y ahorran, empresas que invierten en capital fijo, contratan trabajo y producen bienes y gobierno que

\footnotetext{
3 Además de Bernanke y Gertler y Cecchetti et al., otros trabajos seminales en esta polémica son: Kent y Lowe (1997), Smets (1997), Bank for International Settlements (1998), CEPR (1998), Batini y Nelson (2000), Filardo (2000, 2004), Goodhart (2000a, 2000b), Goodhart y Hoffman (2000), Mishkin (2001) y Bean (2003).
} 
ejecuta política fiscal y monetaria) en el cual consideran los efectos del acelerador financiero -cuyo mecanismo de transmisión afecta el gasto- y de las burbujas en los precios de los activos. Una de las contribuciones principales del modelo Bernanke-Gertler es que los autores analizan el efecto de las burbujas financieras en la hoja de balance (costos de capital, flujos de ingresos y gastos) de las empresas cuando ocurren desviaciones de los precios de los activos respecto de los valores fundamentales, y con base en este análisis infieren la regla de política monetaria óptima que estabiliza la actividad económica y la inflación en presencia de turbulencias en los mercados de activos.

El banco central debe practicar una política monetaria agresiva contra la inflación -medida por el índice de precios al consumidor- a fin de alcanzar el objetivo de estabilización macroeconómica. Al elegir una trayectoria del nivel de precios (objetivo de inflación, $\pi^{*}$ ) la autoridad monetaria minimiza una función de pérdida $\left(L_{b c}\right)$ sujeta a las restricciones impuestas por las leyes dinámicas de la economía. La optimización estabilizadora del banco central se expresa en términos formales de la siguiente manera:

$$
\begin{gathered}
\min E_{t} \sum_{t=0}^{t} \delta^{t} L_{b c} \\
L_{b c}=\left[\pi_{\Gamma}^{2}+\theta\left(y_{\Gamma}^{2}\right)\right]
\end{gathered}
$$

Donde $E_{t}$ es la expectativa matemática en el periodo $t, \delta$ es un factor de descuento de tiempo, $\pi$ es la tasa de inflación, $\pi_{t}$ es la brecha o diferencia entre la inflación observada y la meta de inflación, $y_{\Gamma}$ es la brecha entre el producto real observado y el producto potencial u objetivo o, en el caso de una economía abierta, $y_{\Gamma}$ mide la diferencia entre el tipo de cambio real efectivo y el tipo de cambio objetivo; $\theta$ mide el grado de preferencia por la estabilidad de la inflación con relación a la estabilidad de producto. $\theta=1$ cuando el banco central aplica una regla de objetivo de inflación rígida en una economía de competencia perfecta walrasiana; en cambio, $0<\theta<1$ cuando sigue un enfoque de objetivo de inflación flexible en condiciones de competencia imperfecta caracterizada por precios y salarios rígidos. Sin embargo, la política agresiva contra la inflación no debe comprender una respuesta a las turbulencias y a las burbujas de los precios de los activos porque si, por ejemplo, el banco central reaccionara contra los movimientos de los precios de las acciones de la bolsa de valores podría generar efectos desestabilizadores.

En un contexto de mercados de capitales perfectos, desregulados y eficientes donde prevalece un marco de política monetaria de objetivo de inflación flexible, la estabilidad de precios y la estabilidad financiera son complementarias. Por tanto, el banco central no debe considerar las fluctuaciones de precios de los activos como una meta intermedia, a menos que estas fluctuaciones sean consecuencia de cambios en los fundamentales que modifican las expectativas de inflación o deflación. El efecto riqueza sobre el gasto en consumo que resulta de los movimientos en los precios de los activos no es significativo, como en cambio sí lo es el efecto de estos movimientos de precios sobre las hojas de balance de las corporaciones privadas y sobre la demanda agregada, el empleo y el producto. Estos efectos perniciosos 
pueden redimensionarse a través del acelerador financiero y los mecanismos de deuda-deflación si la contracción de la economía deprime aún más los precios de los activos (Bernanke et al. 2000). Incluso en un contexto de mercados de crédito imperfectos, con fricciones y rigideces, los ajustes de las tasas de interés de un marco de política monetaria IT garantizan que la inestabilidad de los precios de los activos asociados a burbujas no-fundamentales se magnifique hasta recalar en una crisis financiera.

Bernanke y Gertler $(1999,2001)$ analizan un modelo económico en el que el banco central adopta la siguiente regla de política "forward looking":

$$
r_{t}^{n}=\gamma_{\pi} E_{t} \pi_{t+1}+\gamma_{s} s_{t-1}
$$

Donde $r_{t}^{n}$ denota la tasa de interés nominal, $E_{t} \pi_{(t+1)}$ son las expectativas de inflación y $s_{(t-1)}$ es el precio de las acciones bursátiles. Las simulaciones reportadas por Bernanke y Gertler(1999) presentan cuatro reglas de política que incluyen o excluyen reacciones ante choques en los precios de los activos alternativamente. La primera es la regla acomodaticia que ignora los precios de las acciones: $\gamma_{\pi}=1.01, \gamma_{s}=0$; la segunda es una regla agresiva que también excluye los precios de los activos: $\gamma_{\pi}=2, \gamma_{s}=0$; la tercera es acomodaticia y permisiva de los precios de los activos: $\gamma_{\pi}=1.01, \gamma_{s}=0.1 \mathrm{y}$ la última es agresiva y reacciona ante movimientos de los precios de los activos: $\gamma_{\pi}=2, \gamma_{s}=0.1$. Los autores encuentran que la regla de política que mejor estabiliza el producto y la inflación ante un choque en los precios de los activos es la que reacciona agresivamente contra la inflación pero que no reacciona ante burbujas en los mercados financieros. Las políticas que procuran acomodar los choques exógenos en los precios de los activos provocan significativa desestabilización de la inflación y el producto. La disminución del grado de apalancamiento financiero en la economía también contribuye a reducir la volatilidad del producto y de la inflación.

Los resultados de simulación cuantitativa reportados por Bernanke y Gertler (2001:255) muestran que "la superioridad de [la política de] objetivo de inflación agresiva se mantiene para ambos tipos de choques [burbujas de precios de activos y choques tecnológicos] y para la combinación de ambos" con relación a la disminución de la inflación y de la brecha de producto. Bernanke y Gertler consideran que la regla monetaria óptima es la que reacciona ante fluctuaciones en la inflación y en la brecha de producto pero que no incluye los movimientos de los precios de los activos. Admiten que estos movimientos pueden alterar temporalmente la tasa natural de interés, y en ese sentido la política monetaria óptima deberá acomodar esos choques ("otorgar una pequeña ponderación en los precios de las acciones bursátiles por tanto puede ayudar un poco" (Bernanke y Gertler 2001:256). Sin embargo, la mera reacción de la regla de política ante la brecha de producto per se "elimina los beneficios de incluir una reacción ante los precios de las acciones", amén de que es más difícil medir las burbujas y la desviación estándar de los precios de los activos respecto de sus fundamentales (ibid.): "la conducta de la inflación nos da un indicador en tiempo real de la magnitud de la brecha de producto, mientras que no tenemos un indicador análogo que suministre una confirmación de las estimaciones de los fundamentales de las acciones" (ibid.). Además, las simulaciones econométricas de Bernanke y Gertler revelan que los beneficios en términos de la brecha de 
producto que resultan de la inclusión de los movimientos de los precios de los activos en la regla de política son contrarrestados por el incremento en la variación de la inflación. En suma, "concluimos que para valores de parámetros plausibles el banco central no debe responder a los precios de los activos" (ibid.).

En suma, Bernanke y Gertler (1999:78) descartan enfáticamente que los precios de los activos puedan tener un lugar en una regla de política monetaria flexible de inflation targeting: "El enfoque de objetivo de inflación dicta que los bancos centrales deben ajustar la polítca monetaria de manera activa y preventiva para compensar presiones inflacionarias y deflacionarias incipientes. De modo importante, para los propósitos presentes, esto implica también que la política nou debe responder a cambios en los precios de los activos, excepto en los casos en que señalen cambios en la inflación esperada".

Cecchetti et al. (2000, 2002), contrariamente a Bernanke y Gertler, estiman que el banco central sí debe incluir los precios de los activos en su regla de política monetaria. Cecchetti et al. (2000:26) afirman que "la estabilidad macroeconómica sería bien servida si la política monetaria reaccionara en parte a los desequilibrios en los precios de los activos." En un documento posterior (Cecchetti et al. 2002:2) reiteran la esencia de su argumento formulado previamente: "un banco central preocupado por lograr una meta de inflación en un horizonte temporal dado por lograr una trayectoria de la inflación tan suave como sea posible, es muy probable que alcance resultados superiores ajustando sus instrumentos de política no sólo respecto a la inflación ( o a su pronóstico de inflación) y a la brecha de producto, sino también con respecto a los precios de los activos. Típicamente, modificar el marco de política de este modo también podría reducir la volatilidad del producto. Enfatizamos también que esta conclusión se basa en nuestro punto de vista de que reaccionar ante los precios de los activos en el curso de la formulación de política reducirá la probabilidad de que se formen burbujas de precios, reduciendo así el riesgo de ciclos boom-bust de inversión."

Cecchetti et al. (2000) postulan una extensión de la célebre regla de Taylor de la siguiente forma:

$$
r_{t}^{f f}-\pi_{t}=2.5+0.5\left(\pi_{t}-2\right)+0.5\left(y_{t}-y_{t}^{*}\right)+0.05 s_{t-1}
$$

Donde $r_{t}^{f f}$ es la tasa de interés de los fondos federales, $\pi_{t}$ es la inflación rezagada dos años medida por la mediana del índice de precios al consumidor, 2.5 es la tasa de interés real de equilibrio, $\left(y_{t}-y_{t}^{*}\right)$ es la desviación porcentual del PIB observado respecto del PIB potencial y $s_{(t-1)}$ es la magnitud de la burbuja del mercado de valores medida como la desviación porcentual de la inversa de la prima de riesgo presente de las acciones correspondiente a un promedio móvil de veinte años. El modelo de Cecchetti et al. no propone objetivos particulares de los niveles de los precios de los activos ni "targets" para las burbujas de precios de activos, sino la incorporación de los desequilibrios en los precios de los activos en la función de reacción del banco central preservando la estabilidad de los precios al consumidor como el "objetivo primordial del banco central" (Cecchetti et al. 2000:79).

Las estimaciones de Cecchetti et al. se basan en datos de la economía de Estados Unidos abarcando el periodo 1996Q4-1999Q4; se observa que el efecto 
de incluir los precios de los activos en la regla de política aumenta la tasa de interés sistemáticamente por encima tanto de la tasa de interés efectivamente observada como de la implícita en la regla de Taylor convencional, aun considerando una prima de riesgo muy baja, aunque el ejercicio es meramente ilustrativo. Sin embargo, lo esencial del argumento de Cecchetti et al. es que si el banco central responde a las burbujas en los mercados de activos: i) las tasas de interés se moverían "gradualmente en respuesta a las desviaciones de los precios de activos vis-à-vis los fundamentales"; ii) lo anterior permitiría que el incremento requerido en las tasas de interés fuera menor; iii) si bien la estimación de la magnitud de las burbujas y de los desequilibrios en los mercados de activos es compleja, no es más difícil que la estimación cuantitativa de los otros insumos que requiere la regla de Taylor convencional (es decir, la estimación del PIB potencial, la brecha de producto, el tipo de cambio de equilibrio, la tasa natural de interés); iv) dado que la regla de política "forward looking" es preferible a la regla de Taylor simple, la incorporación de pronósticos de los precios de activos es consistente con la regla de política óptima; v) una regla de política que responde anticipadamente a las desviaciones de los precios de los activos respecto de los fundamentales favorece la credibilidad y reputación de la autoridad monetaria porque esta estrategia facilita una estimación ex ante más adecuada de la inflación esperada reduciendo así la volatilidad del producto y de los precios; vi) al incluir la volatilidad de los precios de los activos en su función de reacción, el banco central contribuye a la estabilidad financiera, además de que una política del tipo "leaning against the wind" es más fácil explicar al público (ibid., p. 74); vii) la respuesta de los bancos centrales ante las burbujas en los precios de los activos debe ser simétrica frente a revaluaciones y devaluaciones de las acciones a fin de evitar metas u objetivos del nivel de mercado de valores así como estallidos desestabilizadores de las burbujas especulativas causados por abruptos incrementos de las tasas de interés que generarían descensos recursivos en los precios de las acciones.

Esta estrategia sugiere que las tasas de interés -el instrumento de la política monetaria del banco central- se ajustarían gradual y contra-cíclicamente con relación a la dinámica de los precios de los activos y de acuerdo tanto con los niveles fundamentales cuanto con las expectativas de inflación. Cecchetti et al. sostienen que si su enfoque orientara la regla de política de los bancos centrales, los desequilibrios en los mercados financieros y los ciclos boom-bust en los precios de los activos serían menos drásticos, con cual la autoridad monetaria lograría de modo más eficiente y eficaz la estabilidad macroeconómica.

Cecchetti et al. (2002:18, passim), al evaluar los resultados de la subsecuente simulación estadística de Bernanke y Gertler (2001) y de Batini y Nelson (2000), reiteran la "necesidad de identificar" los movimientos de los precios de los activos justificados y los no justificados por los fundamentales subyacentes y ajustar la función de reacción del banco central en concordancia con ello y concluyen que "si no puedes estimar los desequilibrios de los precios de los activos, tampoco puedes pronosticar la inflación" (ibid., p. 19). 


\section{Balance del debate y una visión alternativa}

¿Las diferencias de enfoque entre Bernanke y Gertler y Batini y Nelson, por un lado, y Cecchetti et al., por otro, son sólo de naturaleza semántica? Cecchetti et al. (2002) opinan que son semánticas en un sentido muy general: "virtualmente todos estamos de acuerdo en que la información contenida en los precios de los activos debe tomarse en cuenta en la medida en que tienen un impacto directo o indirecto en la inflación futura. De aquí que si adoptamos un punto de vista suficientemente amplio respecto de "tienen un impacto en la inflación futura", toda la información relevante ya está incorporada en la decisión de política" (ibid., p. 19).

No obstante el punto de vista conciliador de Cecchetti et al. (2000), Bernanke y Gertler (2001) parecen arribar a una conclusión menos conciliadora porque opinan que la propuesta de Cecchetti et al. es insostenible pues supone que el banco central sabe con certeza que el auge del mercado de valores no se debe a los fundamentales así como el momento de explosión de la burbuja. Así es que al final del debate entre estos autores tenemos dos respuestas dicotómicas a la cuestión de si el banco central debe reaccionar ante la evolución de los precios de los activos. No obstante, en general entre los bancos que siguen una regla de inflation targeting rígida o flexible parece existir un consenso en favor de no reaccionar ante movimientos de los precios de los activos.

En realidad en el debate de marras se tiende a confundir dos cosas que son distintas per se, a saber: la incorporación de los precios de los activos en el objetivo de inflación y la cuestión de cuál es la conducta idónea del banco central frente a movimientos de los precios de los activos. La razón teórica para incluir los precios de los activos en la regla de política estriba en saber en qué medida las variaciones de los precios de los activos reflejan la tasa de inflación futura de bienes y servicios futuros, es decir, la cuestión a determinar es si estos precios suministran un pronóstico superior de la trayectoria futura de los precios de bienes y servicios que el que nos ofrece la regla actual (Filardo 2000). Esta cuestión se torna más compleja aún si se piensa que es posible que el marco de política monetaria de inflation targeting garantice inflación baja sin que por ello necesariamente impida el desarrollo de ciclos boom-bust en los mercados de activos, tal como sucedió en Japón en los años noventa y en Estados Unidos con la crisis Subprime en 2007-2008 y en la periferia de la zona del euro en 2009-2010. La recurrencia frecuente de episodios de "exuberancia irracional" (Shiller 2002) en la era de la liberalización financiera justifica la idea de incluir los precios de los activos en el objetivo de inflación; otro motivo importante es que las autoridades monetarias han permanecido "paralizadas en la práctica" o actúan en forma asimétrica -pasivas ante la gestación de la burbuja y activas en la crisis- cuando se presentan ciclos boom-bust (Goodhart 2001). Por otra parte, la mera incorporación de los precios de los activos en la meta de inflación ya supone que por esa vía obtendremos información más acuciosa sobre el curso futuro de la economía real. Esta es una cuestión práctica y, por tanto, depende de múltiples factores como estructuras de mercado, el desarrollo institucional, el peso del espíritu animal en la conducta de los consumidores e inversionistas, la medida en que la volatilidad de los precios genera ruido que impide discriminar entre riesgo bueno y riesgo malo, etc. La evidencia empírica muestra que la relación entre los movimientos de los precios de activos y el aumento de la 
inflación y el producto es inestable, es decir, los precios de los activos tienen la capacidad de predecir los aumentos en la inflación y el producto en algunos periodos -no siempre- y en algunos países -no en todas las economías (cf. Stock y Watson 2001).

La cuestión normativa de la conducta ideal del banco central ante movimientos en los precios de los activos, como ya hemos visto, desde el punto de vista metodológico ha sido tratada en términos de la habilidad de los economistas para identificar los movimientos que responden a exuberancia irracional y los que obedecen a los fundamentales, entre choques financieros y choques tecnológicos (Cecchetti et al. 2002). La solución de Bernanke y Gertler es una regla de política agresiva sin reacción ante lo que sucede en los mercados de activos, mientras que la de Cecchetti et al. es, al parecer, que el banco central debe intervenir siempre de manera profiláctica para evitar la formación de burbujas, como en los casos de exuberancia racional en Japón en 1989 con los precios de las acciones y la tierra, o el índice NASDAQ en 1999, las acciones Dot.com en 2001 y las hipotecas de bienes raíces en 2007-2008.

La historia muestra que los ciclos booms-busts de los precios de activos no siempre tienen efectos adversos en la economía. En consecuencia no siempre es necesario que el banco central intervenga para minimizar la probabilidad de inestabilidad macroeconómica ni siempre será necesario incluir los precios de los activos en la regla de política. Además, es difícil saber a priori cuándo un aumento en los precios de los activos terminará afectando a la economía real, aunque, desde luego, es deseable que el banco central reaccione cuando la exuberancia irracional causa efectos negativos en el empleo y la actividad económica. Finalmente, en el modelo de política monetaria de la Nueva Síntesis Neoclásica la tasa de interés es el instrumento que el banco central utiliza para conseguir el objetivo de inflación. Sin embargo, la tasa de interés es un instrumento inadecuado y débil para evitar la exuberancia irracional, las burbujas de los precios de los activos y los ciclos boom-bust que causan inestabilidad macroeconómica.

\section{Bibliografía}

Allen, F., and D. Gale (2000). Bubbles and Crises. The Economic Journal, 110, pp. 236-255, January.

Allen, F. and D. Gale (2009). Understanding Financial Crises, Oxford, Oxford University Press.

Arcelli, M. (1996), Telematica Mercati Globali e Derivati Finanziari, en Telema.

Arestis, P., editor (2007). Is There a New Consensus in Macroeconomics?, New York, Palgrave Macmillan.

Ball, L. (1999), Policy Rules for Open Economies, en J. Taylor, editor, Monetary Policy Rules, Chicago, University of Chicago Press.

Bank for International Settlements (1994). Macroeconomic and Monetary Policy Issues Raised by the Growth of Derivatives Markets, Reporte preparado por un grupo de trabajo organizado por el Euro-currency Standing Committee of the central Banks of the Group often countries, Basilea, Suiza.

Bank for International Settlements (1998), The Role of Asset Prices in the Formulation of Monetary Policy, BIS Conference Papers Vol. 5, Basilea.

Bank for International Settlements (2000), Survey of Electronic Money Developments, Working Paper No. 38, Committee on Payment and Settlement Systems, Basilea, Suiza.

Bank for International Settlements (2001), Quarterly Review, Basilea, Suiza, septiembre. 
Batini, N. and E. Nelson (2000), When the Bubble Bursts: Monetary Policy Rules and Foreign exchange Market Behavior?, Mimeo.

Bean, C. (2003). Asset Prices, Financial Imbalances and Monetary Policy: Are Inflation Targets Enough?, Basle, BIS Working Papers no. 140, September.

Bernanke, B. S. and M. Gertler (1990), Financial Fragility and Economic Performance, Quarterly Journal of Economics pag. 105.

Bernanke, B.S. and M. Gertler (1999). Monetary Policy and Asset Price Volatility, en New Challenges for Monetary Policy, Simposium del Federal Reserve of Kansas City, Jackson Hole, Wyoming, agosto 26-28.

Bernanke, B. S., M. Gertler and S. Gilchrist (2000), The Financial accelerator in a Quantitative Business Cycle Framework, en J. Taylor y M. Woodford, editors, Handbook of Macroeconomics, Amsterdam, North-Holland, pp. 1341-1393.

Bernanke, B. S. and M. Gertler (2001). Should Central Banks Respond to Movements in Asset Prices?, American Economic Association Papers and Proceedings, 91(2), pp. 253257, May.

Bernanke, B. S., T. Laubach, F. S. Mishkin and A. Posen (1999). Inflation Targeting: Lessons from the International Experience, Princeton, Princeton University Press.

Bernanke, B. S. and F. S. Mishkin (1997). Inflation Targeting: A New Framework for Monetary Policy?, Journal of Economic Perspectives, 11(2), pp. 97-116.

Bofinger, P. and T. Wollmershäuser (2001). "Managed Floating: Understanding the New International Monetary Order", CEPR Discussion Paper 3064.

Capraro, S. and I. Perrotini (2011), Intervenciones Cambiarias Esterilizadas, Teoría y Evidencia: El Caso de México, Revista de Contaduría y Administración, 57(2), UNAM.

Cecchetti, S. G., H. Genberg, J. Lipski and S. Wadhwani (2000). Asset Prices and Central Bank Policy, Geneva Reports on the World Economy, London, International Center.

Cecchetti, S. G., H. Genberg and S. Wadhwani (2002). Asset Prices in a Flexible Inflation Targeting Framework, Cambridge, MA, NBER Working Papers No. 8970, junio.

CEPR, (1998). Asset Prices and Monetary Policy: Four Views, Londres, Centre for Economic Policy Research for Monetary Policy and Banking Studies and Centre for Economic Policy Research.

Clarida, R., J. Galí and M. Gertler (1997). The Science of Monetary Policy: A New Keynesian Perspective, Journal of Economic Literature, 37(4), pp. 1661-1707.

Cohen, B. (1996). Derivatives and Asset Price Volatility: A Test using Variance Ratios, Working Paper No. 33, Basilea, Bank for International Settlements, enero.

Davis, E. P. (1995), Debt Financial Fragility and Systemic Risk, Oxford, Oxford University Press.

Dewatripont, M., J. C. Rochet, and J. Tirole (2010), Introduction, Balancing the Banks, Princeton Princeton University Press, 1-9.

Duménil, G. and D. Levy (2011). The Crisis of Neoliberalism, Cambridge, MA, Harvard University Press.

Federal Reserve (2012), Fedwire Funds Service Annual Data, disponible en: http://www.federalreserve.gov/paymentsystems/fedfunds_ann.htm.

Filardo, A. J. (2000), Monetary Policy and Asset Prices, Federal Reserve Bank of Kansas City Economic Review, Third Quarter, 85(3), pp. 11-37.

Filardo, A. (2004). Monetary Policy and Asset Price Bubbles: Calibrating the Monetary Policy Trade-offs, Basle, BIS Working Papers no. 155, June.

Frenkel, M.; C. Pierdzioch; G. Stadtmann (2003), The Effects of Japanese Foreign Exchange Market Interventions on the Yen/U.S. dollar Exchange Rate Volatility, Kiel WP No. 1165, Mayo.

Fullenkamp, C.R. and S. Nsouli (2004). Six Puzzles in Electronic Money and Banking, IMF Working Paper No. 04-19, Washington D.C. IMF.

Goodhart, C. A. E. (2000a), Can Central Banking survive the IT Revolution?, International Finance 3(2), 189-209.

Goodhart, C. A. E. (2000b), Asset Prices and the Conduct of Monetary Policy, Mimeo. 
102 Nueva Época REMEF (The Mexican Journal of Economics and Finance)

Goodhart, C. A. E. (2001). What Weight should be Given to Asset Prices in the Measurement of Inflation?, The Economic Journal, 111, pp. 335-356, June.

Goodhart, C. A. E. and B. Hoffman (2000), Do Asset Price help to Predict Consumer Price Inflation?, Mimeo.

Guttmann, R. (2003), Cybercash: The Coming Era of Electronic Money, Basingstoke, R.U., Palgrave Macmillan.

Hernández Trillo, F. (2003), La Economía de la Deuda: Lecciones desde México, México, Fondo de Cultura Económica.

Hüfner, F. (2004). Foreign Exchange Intervention as a Monetary Policy Instrument, Mannhein, Center for European Economic Research, 175.

Kane, E. J. (1991), Financial Regulation and Market Forces, Swiss Journal of Economics and Statistics, Julio.

Kent, Ch. and Ph. Lowe (1997). Asset-Price Bubbles and Monetary Policy, Research Discussion Paper 9709, Economic Research Department, Reserve Bank of Australia.

Mántey, G. (2009). Intervención esterilizada en el mercado de cambios en un régimen de metras de inflación: la experiencia de México, Investigación Económica, vol. LXVIII, número especial, 47-78.

Mishkin, F. S. (2001). The Transmission Mechanism and the Role of Asset Process in Monetary Policy, Cambridge, MA, NBER Working Paper 8617, available at http://www.nber.org/papers/w8617.

Orhangazi, Ö. (2008). Financialization and the US Economy, Cheltenham, UK.

Shiller, R. J. (2002), From Efficient Market Theory to Behavioral Finance, Cowles Discussion Paper no. 1358, Yale University.

Smets, F. (1997), Financial Asset Prices and Monetary Policy: Theory and Evidence, CEPR Working Paper No. 1751, noviembre.

Stock, J. and M. Watson. (2001). Forecasting Output and Inflation: The Role of Asset Prices, NBER Working Paper no. 8180.

Svensson, L. E. (1998). Inflation Forecast Targeting: Implementing and Monitoring's Inflation Targets", European Economic Review, 46, pp. 771-80.

Svensson, L. E. (1999). Open-Economy Inflation Targeting, Journal of International Economics.

Taylor, J. B. (1993). Discretion versus Policy Rules in Practice, Carnegie Rochester Conference, Series on Public Policy 39, pp. 195.214.

Tirole, J. (2010). Lessons from the Crisis, en Dewatripont, M., J. C. Rochet, y J. Tirole (2010), "Introduction", Balancing the Banks, Princeton, Princeton University Press, pp. 10-77.

Vrolijk, C. (1997). Derivatives effect on Monetary Policy Transmissions, Washington, IMF Working Paper, septiembre.

Woodford, M. (2003). Interest and Prices, Princeton, Princeton University Press. 